\title{
Experimental Test on Bridge Reinforcement by Enlarging Section-Prestress Method
}

\author{
Li Jia ${ }^{1, *}$, Liu Tao ${ }^{2,3}$, Huang Hong ${ }^{2}$, Jiang Jian ${ }^{1}$, Huang Zhi $^{2}$ \\ ${ }^{1}$ Shenzhen Tagen $<$ Group $>$ Co., Ltd,. 518034 Shenzhen, China \\ ${ }^{2}$ Shenzhen Yuetong Construction Engineering Co., Ltd,. 518019 Shenzhen, China \\ ${ }^{3}$ Shenzhen Tagen Engineering Technology Co., Ltd, 518034 Shenzhen, China
}

\begin{abstract}
This paper proposes the method of enlarging section combined with prestressed tendon (Enlarging Section-Prestress Method), which has the advantages of both increasing section and external prestressing, such as reducing section height, the prestressing tendon do not rust easily, and the appearance is beautiful. Model tests were carried out, two rectangular beam models with the same section and length were made, one was reinforced by Enlarging Section-Prestress Method, and no reinforced action was taken as a control. Two points of concentrated load were applied to the model beams, and the two tests were compared in terms of deflection-load characteristics and crack development. The experimental results show that the Enlarging Section-Prestress Method is effective, which improved the ultimate bearing capacity of the beam obviously, and reduced the deflection of the trabecular beam.
\end{abstract}

\section{Introduction}

Once upon a time, bridge design and construction focused on material saving, resulting in low safety stock of bridge structure. With the rapid development of economy, the traffic volume and vehicle load carried by the bridge have increased significantly. In addition, the durability defects of traditional materials also cause the normal aging of the bridge, resulting in certain damage of the safety of the bridge which has operated for many years. In recent years, continuous bridge safety accidents make people realize the severity of bridge reinforcement.

In recent years, the proportion of damaged bridges in China is on the rise. Taking Shenzhen as an example, in 2018, 112 of 957 bridges are rated as grade $\mathrm{C}$ or below in the regular inspection of highways, bridges and culverts, accounting for about $11.7 \% \%^{[1]}$. With the continuous operation of existing bridges, there will be more bridges with different degrees of damage in the future. Building new bridge not only needs a huge investment, but also has a long-term impact on the traffic environment. Therefore, reinforcing the damaged bridge has significant social and economic benefits.

\section{Enlarging Section-Prestress Method}

Reinforced concrete and prestressed concrete simplesupported beam-bridge is the most commonly used bridge structure. The cross-section forms include rectangle, T-shaped, I-shaped, box-shaped and other different combinations. In the early stage, cast-in-place construction was adopted, and now most of them are prefabricated assembly.
The bending moment of the simple-supported beambridge in the middle of the span is the largest, so the bottom of the beam near the middle of the span often produces vertical bending cracks from the bottom to the top, the number of which increases with the increase of the span. The width of cracks for many years' operation may exceed the limit value of the specification, and at the same time, the deflection in the middle of the span may be too large.

The method of enlarging section ${ }^{[2]}$ for reinforcement has advantages of strong adaptability, simple construction technology, mature design and construction experience, and is widely used in various structures such as factory buildings, bridges, wharves, etc. Yang Ye et al. ${ }^{[3]}$ applied the planting-bar technique to the new and old joint surface of concrete, forming an improved method of enlarging section for reinforcement. Liu Lixian et al. ${ }^{[4 \sim 5]}$ of Tsinghua University put forward a simplified calculation method of enlarging section to strengthen the ultimate bearing capacity of reinforced concrete beams with high-temperature damage, and verified through experiments that the bearing capacity and rigidity of columns with high-temperature damage strengthened by this method are greatly improved compared with those before reinforcement. However, this method will reduce the clearance of the strengthened structure and affect the use of the structure. Using external prestressing reinforcement of adding prestressing tendons at the bottom of the beam can reduce the increase of the section height of the component and reduce the impact on the use function of the structure, but the exposed prestressing tendons are easy to corrode and unsightly ${ }^{[6]}$.

\footnotetext{
* Corresponding author: li_jia@tju.edu.cn
} 
In this paper, the reinforcement method of enlarging section combined with prestressed steel strand (Enlarging Section-Prestress Method) is proposed, which combines the advantages of both enlarging section and external prestressing, reducing the increase of section height of the method of enlarging section, making the steel strand not easy to corrode, and ensuring the beauty of the structure.

\section{Model test}

\subsection{Model design and production}

Two model beams were made in the experiment, one of which was strengthened by the Enlarging Section-
Prestress Method, and the other was not strengthened for comparison.

The prestressed concrete beam model is $5400 \mathrm{~mm}$ long, the size of steel bar and section is as shown in Fig.1, the concrete strength grade is $\mathrm{C} 40$, and the thickness of the protective layer of the steel bar is $65 \mathrm{~mm}$. The prestress is applied to three cold-drawn steel bars in the tension area, with the stress $\sigma$ con $=400 \mathrm{MPa}$, and the model components are maintained under natural conditions. The reinforced part is $200 \mathrm{~mm}$ high and 3400 $\mathrm{mm}$ long. The original beam and the strengthening beam are connected by roughening and planting bars, and three steel strand channels are reserved.

The finished model is shown in Fig.2.

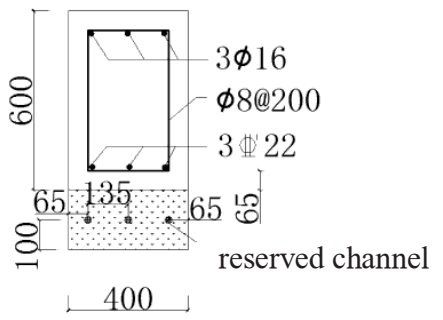

(a)transection

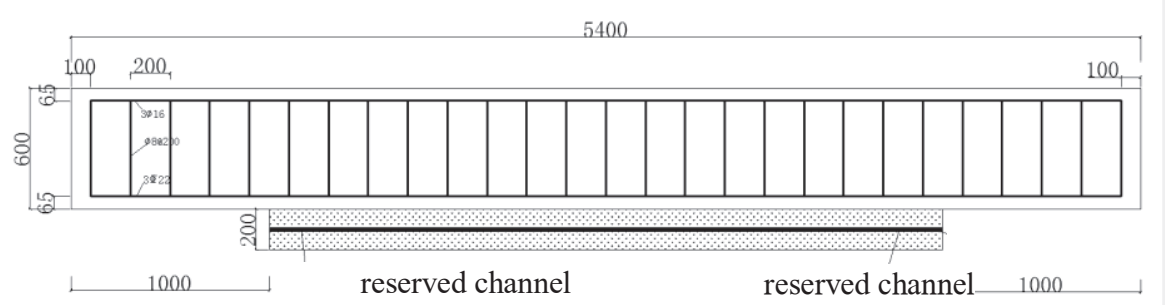

(b) side

Fig.1 the prestressed concrete beam model

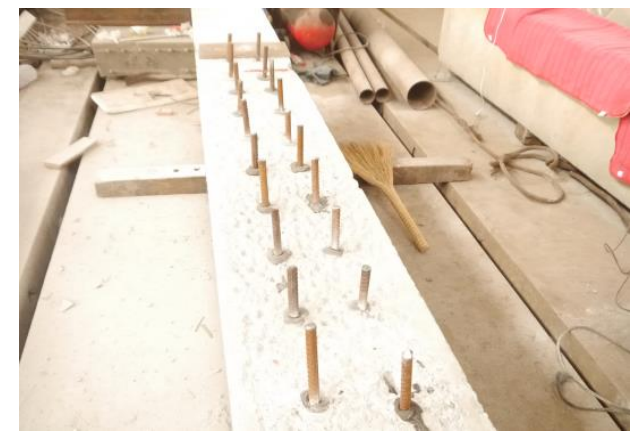

Fig.2 model making

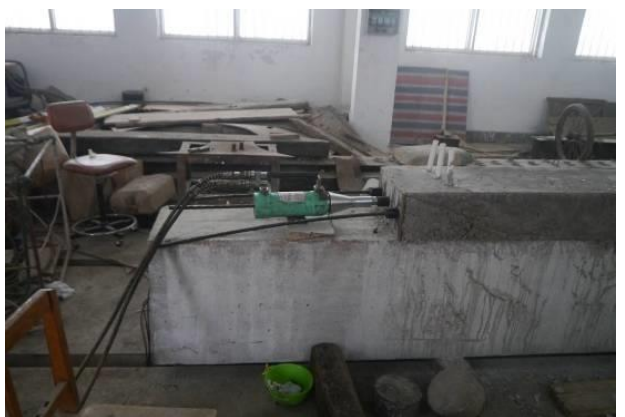

Fig.3 application of prestressing force

\subsection{Design and application of prestress}

The $\Phi 15.2 \mathrm{~mm}$ steel strand is adopted, and the tension control stress of the steel strand is calculated and determined according to Specification for Design of Highway and Bridges Reinforcement JTC/T J22-2008, that is, $\sigma$ con, $\mathrm{e} \leq 0.65 \mathrm{fpk}$,e, and the prestress loss is calculated according to the Specification. The final tensile stress is determined as $875 \mathrm{Mpa}$.

Each steel strand is tensioned twice by using the OVM 240Q jack. The tensioning process and anchoring after tensioning are shown in Fig.3.

\subsection{Test scheme}

The model is supported on the pedestal which is supported on the buttress. The vertical concentrated load is applied by two jacks located at the left and right $1 / 3$ of the span respectively. The reaction force of the jacks is provided by the reaction rigid frame test bench. Five dial indicators are arranged to measure the deflection of the beam during the loading process. Indicator 1 and 5 are respectively located on the top surface of the beam above the left and right pedestals, indicator 2 and indicator 4 are respectively located on the top surface of the beam outside the left and right loading points, $20 \mathrm{~cm}$ from the loading jack, and indicator 3 is located on the top surface of the beam in the middle of the span. The layout of loading and testing device is shown in Fig.4, and the test loading condition is shown in Fig.5.

In the test, preload shall be carried out first to check whether the pedestal is stable and whether the instrument and loading equipment are normal. Then the instrument 
and equipment should have zero-setting. During preloading, the deformation of specimen shall be controlled within the elastic stress range, and no cracks or other loading residual values shall be produced. According to the theoretical calculation, the value of the concentrated force corresponding to the ultimate bearing state of the beam is $144 \mathrm{kN}$, so the loading capacity of each stage is taken as $10 \mathrm{kN}$, and the holding time is taken as 10min. During the loading process, pay attention to whether the instrument is normal and whether the concrete beam is cracked.

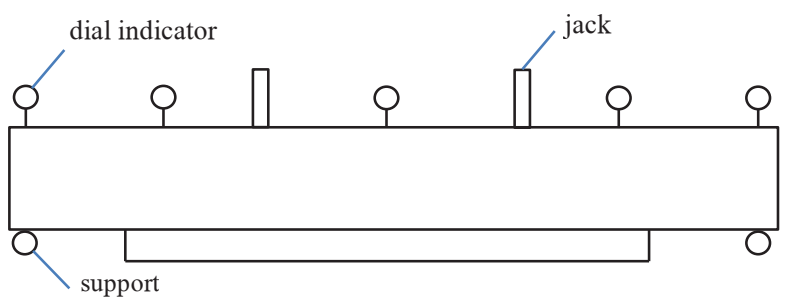

Fig.4 the setup of load and test equipment

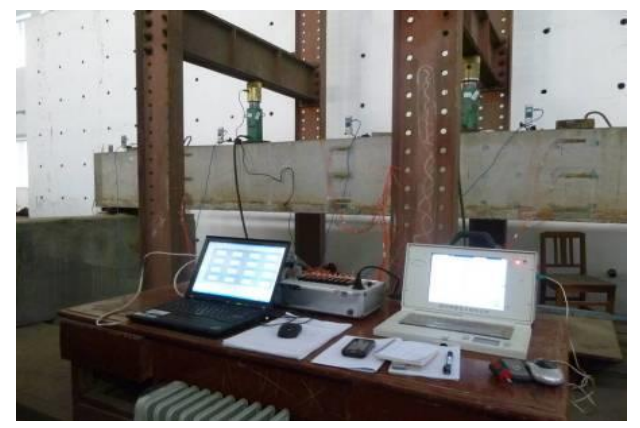

Fig.5 loading in test

\subsection{Test phenomenon}

In the test without reinforcement measures, when the load reaches $110 \mathrm{kN}$, there are cracks at the bottom of the beam. When the load reaches $130 \mathrm{kN}$, there are five cracks between the two loading points, with a length of about $20 \mathrm{~cm}$, evenly distributed, and an interval of about $30 \mathrm{~cm}$. After loading to $140 \mathrm{kN}$, the cracks continue to develop, but the crack width does not increase significantly, and new cracks appear outside the two loading points. When loading to $150 \mathrm{kN}$, the crack length increases slightly, but the crack width increases obviously at the bottom of the beam. When the load reaches $156 \mathrm{kN}$, the crack length and width both increase sharply, and new cracks appear outside the two loading points. In addition, the jack load increases slowly, the dial indicator reading increases sharply, and the "crackle" sound of concrete cracking can be heard obviously. The increase of deflection of concrete beam in the middle of span can be observed by naked eyes. At this time, the crack width is very large, and develops to less than $10 \mathrm{~cm}$ from the beam top. See Fig.6(a) and Fig.7(a) for test photos and crack development condition.

In the test with reinforcement measures, when the load is $110 \mathrm{kN}$, three cracks appear in the reinforcement area, but the original beam area appears nothing. Continue to load to $160 \mathrm{kN}$, and cracks appear in the original beam, mainly distributed at the bottom of the beam corresponding to the loading point. As the loading continues, the cracks continue to develop, and new cracks appear, which are still mainly concentrated near the loading point corresponding to the beam bottom. When the load reaches $200 \mathrm{kN}$, the end of the reinforcement area is separated from the contact part of the original beam, and the length of the separation area is about $10 \mathrm{~cm}$. When the load reaches $240 \mathrm{kN}$, the length of the separation between the end of the reinforcement area and the contact part of the original beam has reached more than $40 \mathrm{~cm}$. The reinforcement area is mainly connected with the original beam by planting bars, but when the load reaches $240 \mathrm{kN}$, there are several oblique cracks at the end of the original beam where the bars are planted. The crack width is relatively large and it is a through crack. The deflection of the beam increases sharply when the jack continues to be pressurized, but the load sensor reading is maintained at $240 \mathrm{kN}$ all the time. Therefore, the ultimate bearing capacity of the beam strengthened by this method is considered to be 240kN. See Fig.6(b) and Fig.7(b) for test photos and crack development condition.

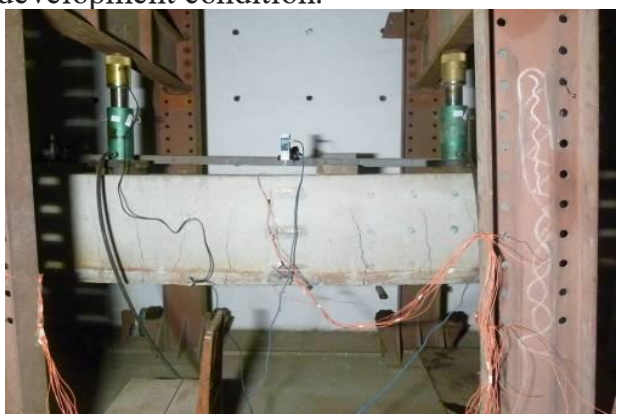

(a) no reinforcement measures

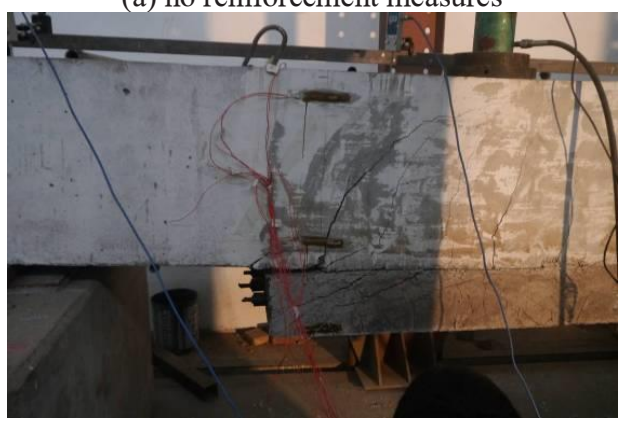

(b) reinforcement by enlarging section-prestress method

Fig.6 beam body during loading test 


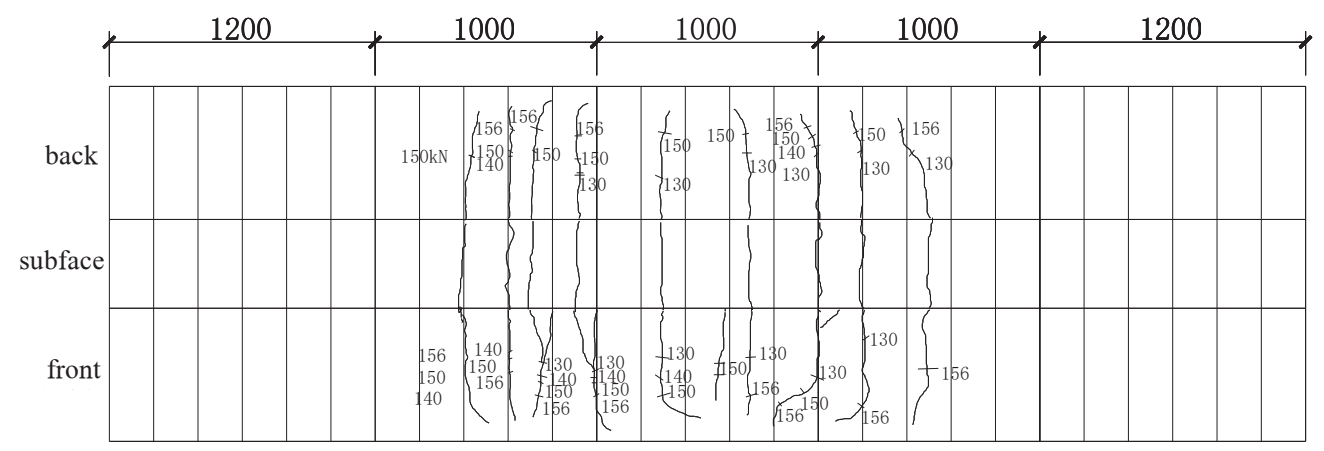

(a) no reinforcement measures

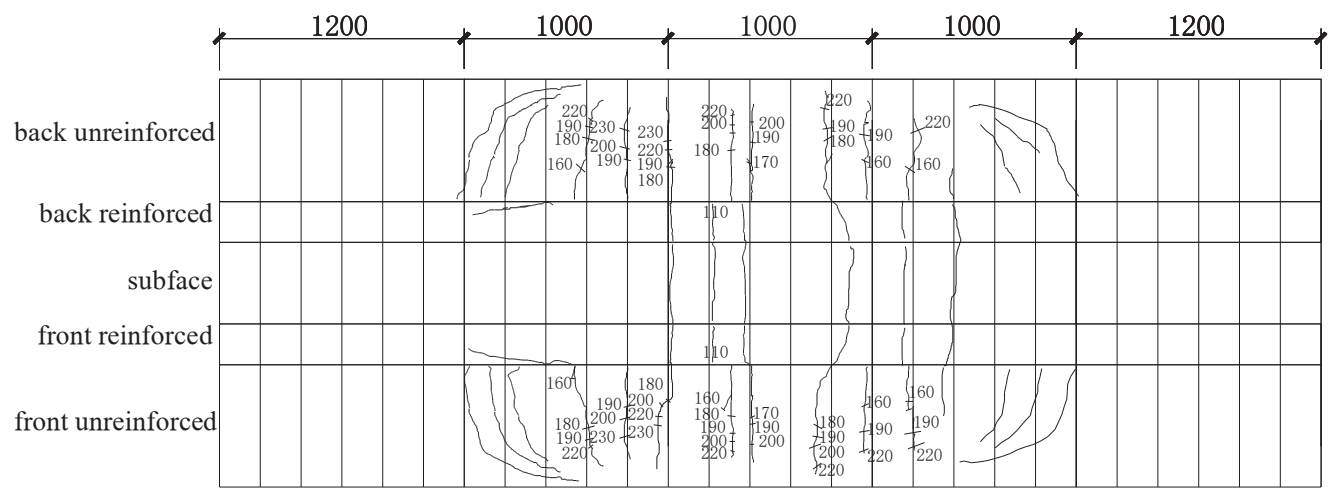

(b) reinforcement by enlarging section-prestress method

Fig.7 crack development during beam failure

\section{Test result}

No. 1 and No.5 dial indicators are located above the pedestal, and there is no change in reading during the test. The load-deflection curve of No.2, No.3 and No.4 dial indicators is shown in Fig.8.

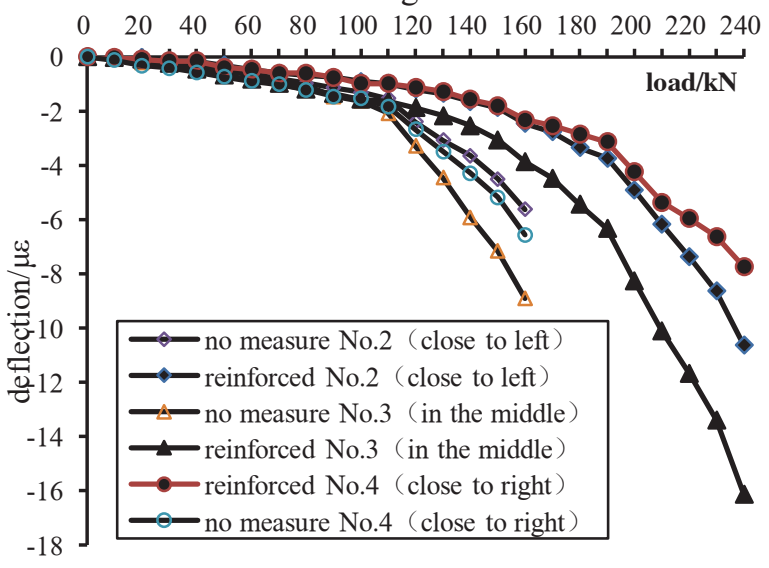

Fig.8 the load-deflection contrast between with or without reinforcement

For beam model without reinforcement measures, when the applied load reaches $160 \mathrm{kN}$, which is ultimate bearing capacity, the deflection increases sharply. At this time, the deflection of the strengthened beams is still very small and just enters the nonlinear stage.

Before the crack occurs in the reinforcement area (i.e. when the load is less than $110 \mathrm{kN}$ ), the deflection of the strengthened beam with Enlarging Section-Prestress Method basically increases linearly and the value is not large; after the crack occurs in the reinforcement area and the original beam does not crack (i.e. the load is between $110 \mathrm{kN}$ and $160 \mathrm{kN}$ ), the deflection increases with the increase of the load; when the original beam cracks, the deflection increases sharply with the increase of the load.

\section{Conclusion}

The following conclusions can be drawn by comparing the static test results of the model beam strengthened by the Enlarging Section-Prestress Method with that of the model beam without reinforcement measures.

(1)The strengthening effect of the Enlarging SectionPrestress Method is obvious, and the ultimate bearing capacity of the beam is increased by $50 \%$.

(2)After strengthening the beam with the Enlarging Section-Prestress Method, the section inertia moment of the beam is increased and the deflection of the beam is reduced obviously.

(3)The prestressing force applied by the steel strand in the reinforcement area has a reverse arch effect on the beam, which also reduces the deflection of the beam to a certain extent.

(4)The end of the reinforcement area is easy to be separated from the contact part of the original beam, so it is recommended to properly densify the reinforcement part when the planting-bar method is used.

In a word, the Enlarging Section-Prestress Method has the advantages of both enlarging section method and external prestressed reinforcement. The test results show that the method has a good reinforcement effect, and less 
impact on bridge clearance than the method of simply enlarging section, which has a certain promotion value.

\section{References}

1. Transport Committee of Shenzhen Municipality, General report on regular inspection of highways bridges and culverts in 2018.(2018).

2. Lin Ningdong. An Example of Strengthening the Structure for a Mill Building with Oversize Section Method. .Industrial Construction, 35 (2005)

3. Yang Ye, Zhao Laishun, Zhao Man. Experimental Investigation of Axial Compression Column Be Strengthened with the Method of Enlarging Section Areas, Building Structure, 06 (2010)

4. Liu Lixian, Shi Xudong, Guo Zhenhai. Simplified calculation on ultimate load of strengthened reinforced concrete flexural members exposed to high temperature, Journal of Southeast University (Natural Science Edition). 32 (2002)

5. Liu Lixian, Shi Xudong, Guo Zhenhai. Experimental investigation of strengthened reinforced concrete columns after exposure to high temperature, Engineering Mechanics, 20 (2003)

6. Zhu Zhengwei, Liu Dongyan, Peng Wenxuan. Consideration of external prestressing technique applied to bridge reinforcement, Journal of Chongqing Jianzhu University, 27 (2005) 